\title{
Annotations to the Devonian Correlation Table, B 144-145 dm-ds 06: Stromatoporoid and Tabulate Coral biostratigraphy in the Middle and Upper Devonian of the Ferques area, Boulonnais (North of France).
}

\author{
Bruno MistiaEN
}

In the Boulonnais (Palaeozoic inlier of Ferques), the Devonian transgression did not reach the area before Middle Devonian times (Givetian probably). Six lithostratigraphic formations, alternating siliciclastic and carbonate, have been recognized (BRICE et al. 1979), from bottom to top: Caffiers, Blacourt, Beaulieu, Ferques, Hydrequent and Sainte Godeleine Formations.

Stromatoporoids and tabulate corals are well represented in the carbonate formations i.e. the Blacourt (Givetian) and the Ferques (Frasnian) formations. However, some species are also present in smaller limestone levels intercalated in the Beaulieu Formation (Frasnian), especially in the Noces Member.

Moreover, the stromatoporoids occur most commonly in the carbonate facies and are particularly numerous in the Griset Member of the Blacourt Formation (Givetian), where 13 stromatoporoid levels have been recognized (Mist iaen, 1988a).

The stromatoporoid biodiversity is clearly more important in the Givetian (42 taxa) than in the Frasnian (18 taxa); this is not so obvious for the Tabulate corals.

In the correlation tables, the total distribution, from the first to the last occurrence, is represented for each species.

\section{References}

Brice, D., Bigey, F., Mistiaen, B., Poncet, J. \& Rohart, J.Cl. (1977): Les organismes constructeurs (Algues, Stromatopores, Rugueux, Tabulés, Bryozoaires) dans le Dévonien de Ferques (Boulonnais - France) Associations - Répartition stratigraphique. - Mémoire du Bureau des Recherches géologiques et minières, 89: 136-151; Paris.

Brice, D., Bultynck, P., Colbeaux, J.P., Lethiers, F., Mistiaen, B., RoHART, J.Cl. \& BigeY, F. (1976): Une nouvelle coupe dans le Dévonien de Ferques (Boulonnais, France). - Annales de la Société géologique du Nord, 96 (2): 135-155; Lille.
Brice, D., Colbeaux, J.P., Mistiaen, B. \& Rohart, J.Cl. (1979): Les Formations dévoniennes de Ferques (Bas-Boulonnais, France). - Annales de la Société géologique du Nord, 98: 307-324; Lille.

Brice, D., Milhau, B., Mistiaen, B., Rohart, J.Cl. \& Vidier, J.P. (1989): Le Givétien supérieur (Dévonien) à Ferques (Boulonnais - France) Observations nouvelles. - Annales de la Société géologique du Nord, 108: 113-123; Lille.

Brice, D., Mistlaen, B. \& Rohart, J.Cl. (2002): Progrès dans la connaissance des flores et des faunes dévoniennes du Boulonnais (1971-2001). - Annales de la Société géologique du Nord, 9 (2 $2^{\text {ème }}$ série): 61-74; Lille.

Hung, N.H. \& MistiaEN, B. (1997): Vacuustroma, un genre nouveau de stromatopore du Dévonien du Vietnam et du Boulonnais (France). - Geobios, 30 (2): 193-204.

Mistiaen, B. (1988a): Stromatopores du Givétien et du Frasnien de Ferques (Boulonnais - France) - In: Brice D. (éd.), Le Dévonien de Ferques, Bas-Boulonnais (N. France) Biostratigraphie du Paléozoïque, Brest, 7: 163-195.

Mistiaen, B. (1988b): Tabulés Auloporida du Givétien et du Frasnien de Ferques (Boulonnais - France) - In: Brice D. (éd.), Le Dévonien de Ferques, Bas-Boulonnais (N. France) Biostratigraphie du Paléozoïque, 7: 197-230; Brest.

Mistiaen, B. (1988c): Dendropora explicita MicheLIN 1846 (Tabulata) dans sa localité type, le Boulonnais. $12^{\mathrm{eme}}$ Réunion RST, Lille Société Géologique de France éd.: 98.

Mistiaen, B. (1990): Dendropora explicita Michelin, 1846 et $D$. briceae nov. sp. (Tabulata) dans leur localité-type du Boulonnais. Présence du genre en Afghanistan. - Geobios, 24 (2): 141-155; Lyon.

Mistiaen, B. (2002): Stromatopores et Coraux tabulés du Membre des Pâtures, Formation de Beaulieu (Frasnien de Ferques, Boulonnais). - Annales de la Société géologique du Nord, 9 ( $2^{\text {eme }}$ sér): 85-90; Lille.

Mistiaen, B., Becker, R.T., Brice, D., Degardin, J.M., Derycke, Cl., LoONes, CH. \& RoharT, J.Cl. (2002): Données nouvelles sur la partie supérieure de la Formation de Beaulieu (Frasnien de Ferques, Boulonnais, France). - Annales de la Société géologique du Nord, 9 (2 $2^{\text {ème }}$ sér): 75-84, Lille. 